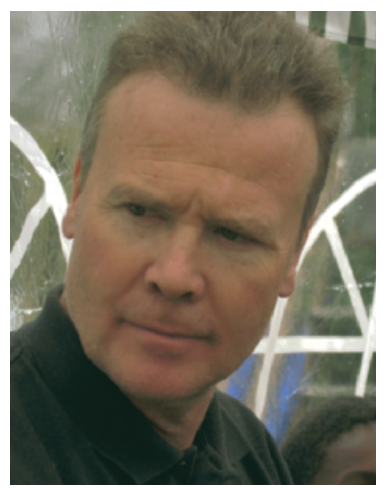

G. Dine

\section{Une biologie clinique pour tous les âges}

A l'occasion de ce numéro, un dossier spécial a été développé sur la relation entre biologie médicale et gériatrie. Le vieillissement de la population française est un fait inéluctable. Cette situation impacte les professionnels de santé quels qu'ils soient. Elle modifie également l'organisation de notre société et, question d'actualité oblige, le financement des différentes prises en charge et solidarités pour les individus entrant dans cette période de vie. Les marqueurs biologiques n'échappent pas à cette réalité. La définition de valeurs référentes biologiques adaptées aux catégories d'âges est nécessaire. Vis-à-vis des besoins gériatriques, on pourrait même parler de plusieurs âges, vu les segmentations qui apparaissent au sein des populations concernées. L'organisation de prélèvements plus ambulatoires et, par conséquent, les phases pré-analytiques doivent être absolument réfléchies afin de pouvoir traiter les échantillons d'une façon correcte et fiable une fois rendus aux laboratoires d'analyses. L'utilisation de technologies disponibles au lit du patient ou à son domicile doit être envisagée même si ces outils échappent au cadre hospitalier. Dans ces conditions, la logistique biomédicale va jouer un rôle certain. La centralisation et la validation de ces résultats recueillis par les systèmes d'information qui utiliseront les différentes connectiques actuelles, doivent être envisagées communément par les biologistes et les cliniciens concernés. La participation des diverses catégories de professionnels de santé sera donc indispensable. Leur coordination sera un gage d'efficacité et de fiabilité. L'interprétation des données biologiques issues de populations âgées nécessite une compréhension particulière qui est développée dans ce dossier. La relation entre résultats biologiques et décisions médicales doit être absolument établie et reliée à la particularité des personnes âgées. Certaines limites à la validité d'un test existent. Plusieurs contraintes quant à la pertinence d'un marqueur biologique sont réelles. Le déclenchement de l'indication à traiter ou hospitaliser à partir du résultat biologique sous-entend la bonne intégration de ces spécificités propres à la personne âgée. La relation à l'environnement du patient en termes de surveillance, d'intervention et d'autonomie ne peut pas ignorée. On le voit, le biologiste n'échappera pas à la réflexion qu'il doit mener sur les corrélations à établir entre résultats biologiques et personnes âgées mais le biologiste devra aussi prendre en compte les phases pré-analytiques et les situations environnementales propres à ces patients. Le rôle du biologiste médical du point de vue de l'interprétation et des conseils à donner au clinicien pour décision ne sera que valorisé mais il sera plus conséquent en matière d'expertise. • 